\title{
Micro X-ray Fluorescence: A personal perspective of 30 years
}

\author{
George J. Havrilla ${ }^{1}$
}

1. Los Alamos National Laboratory, Los Alamos, NM 87545 USA.

As an analytical scientist charged with providing solutions to problems based on the elemental characterization of samples provided to me, I have found over the years that MXRF (micro X-ray fluorescence) offered a unique and rapid capability which has developed and evolved over the years into a very powerful and useful technique for solving these myriad of analytical problems. I have witnessed the evolution of the instrument from a basic collection of excitation source and detector to a refined instrument capable of creating exquisite elemental maps with sub-10 micrometer spatial resolution covering $\mathrm{mm}$ to $\mathrm{cm}$ sized areas all within the confines of a laboratory-benchtop. This presentation will highlight the MXRF instrument developments in X-ray tube sources and detectors as well as the optics which propelled MXRF into the mainstream analytical toolbox. My efforts over the last 30 years has been one of educating people unaware of the capabilities of MXRF and expounding on the benefits and advantages of MXRF in solving analytical problems encountered in my lab.

Laboratory-based MXRF first appeared in the early 1960's, however it was soon eclipsed by the use of electron-based X-ray characterization with much higher spatial resolution. [1] It was in the late 80's and 90's when commercial instruments such as the Kevex Omicron became available [2]. These early instruments relied on apertures or glass capillaries to create a spatially resolved X-ray probe beam to obtain spatially resolved elemental spectra from samples of interest. My first experience with MXRF was an EDAX MAX unit, which was essentially a cast steel chamber with the X-ray tube and SiLi detector bolted to the chamber at 45 degrees to the sample which resided on an $\mathrm{x}, \mathrm{y}, \mathrm{z}$ stage manually adjusting the position with micrometers. The X-ray tube was equipped with several apertures which screwed into the tip of the tube housing to provide 'spatial resolution' from 1-3 mm. Although a crude assembly, it worked well enough to obtain spatially resolved point spectra from a variety of specimens and solve some analytical problems, clearly demonstrating the usefulness of spatially resolved XRF.

The big advance in the field was the commercial appearance of the Kevex Omicron, shown in Figure 1, with its mapping capabilities and easily interchanged apertures ranging from 50 micrometers to 1000 micrometers. The photon flux arriving at the specimen with the 100 micrometer aperture strained the patience of the instrument operator in accumulating sufficient elemental signals to create elemental distribution maps. However, the advent of the Omicron signaled the development of MXRF as a laboratory technique for creating elemental maps covering large areas from $\mathrm{mm}^{2}$ to multiple $\mathrm{cm}^{2}$. Figure 2 shows some of the early work using the Omicron in obtaining 3 different types of elemental information: 1) single point mode, 2) line scans and 3) elemental maps. In addition to the ability to obtain different modes of elemental information, the SiLi energy dispersive detector allowed for rough surfaces, nonconductive samples, operation at atmosphere and with helium purge and it was nondestructive. All of these features helped propel MXRF into the mainstream toolbox of the analytical chemist trying to solve characterization problems.

The development of actual X-ray optics to focus X-rays to 10-50 micrometer spot sizes in the form of monolithic polycapillaries, transformed MXRF from a quietly developing analytical method into a technique with significant sensitivity and ppm to sub-ppm detection limits surpassing conventional bulk 
XRF technologies.[3] The incorporation of polycapillary optics into commercial instrumentation created a modest increase in commercial instruments providing unique capabilities to materials characterization and analytical problem solving. The ability to achieve 10 or even sub-10 micrometer spatial resolution opens up capabilities which can now probe significant materials questions with regards to particle characterization and studies of biological significance, since these X-ray probe spot sizes are on the order of cells, tissues and particle sizes of interest. A new capability was developed by adding a polycapillary in front of the detector to create a confocal MXRF instrument. This has unique nondestructive 3D elemental analysis capabilities for sub-surface elemental mapping.

In addition to the polycapillary optics, doubly curved crystals (DCCs) have made an impact on MXRF by providing point-to-point focusing as well as monochromatic beam conditioning resulting in low background and high signal-to-noise capabilities. [4] Incorporating a DCC on the excitation probe and another DCC as a collection optic prior to the detector creates a monochromatic wavelength dispersive $\mathrm{XRF}$ instrument which can routinely provide sub-ppm sensitivity for quantitative elemental analyses and mapping.

The future of MXRF appears bright with the advent of silicon drift detectors (SDDs) which increase the maximum count rate abilities and can now fully take advantage of the high flux from the polycapillaries and DCCs. In addition, to these detectors, microcalorimeter detectors, offer 1-10 eV spectral resolution which enables not only elemental characterization but characterization of the oxidation state of the element as well.

\section{References :}

[1] Adler, I., Axelrod, J., and Branco, J. J. R., Adv. X-ray Anal. 5 (1962) p.2.

[2] Wherry, D. C., Cross, B. J., and Briggs, T. H. Adv. X-ray Anal. 31 (1988) p.93.

[3] Gao, N., Ponomrev, I., Xiao, Q. F., Gibson, W. M., and Carpenter, D. A. Appl. Phys. Lett., 71 (1998) p.3441.

[4] Chen, Z., Gibson, W. M., Powder Diffraction 17 (2002) p. 99.

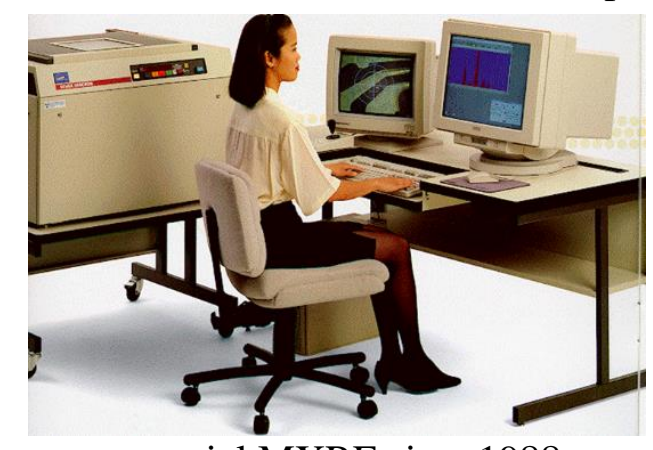

Figure 1. Kevex Omicron commercial MXRF circa 1988.
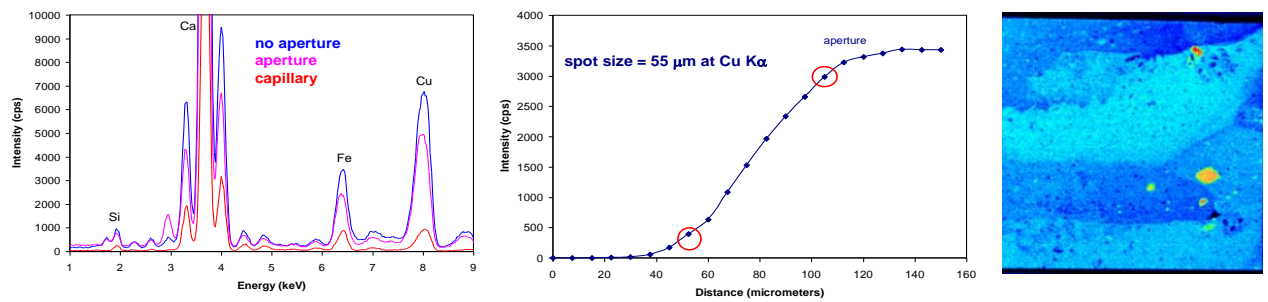

Figure 2. Examples of single point spectra, line scan and elemental map capabilities of MXRF. 